\title{
Shape analysis of wavelength-insensitive grating in the resonance domain
}

\author{
Tetsuya Hoshino $^{\mathrm{a}, *}$, Saswatee Banerjee ${ }^{\mathrm{a}}$, James B. Cole ${ }^{\mathrm{b}}$, Masahide Itoh ${ }^{\mathrm{a}}$,Toyohiko Yatagai ${ }^{\mathrm{a}, \mathrm{c}}$ \\ Institute of Applied Physics, University of Tsukuba, 1-1-1 Tennoudai, Tsukuba 305-8577, Japan \\ ${ }^{b}$ Department of Systems and Information Engineering, University of Tsukuba, 1-1-1 Tennoudai, Tsukuba 305-8577, Japan \\ 'Center for Optical Research and Education, Utsunomiya University, 7-1-2 Yoto,Utsunomiya, Tochigi 321-8585, Japan
}

\begin{abstract}
A triangular grating was compared with a rectangular one to investigate the effect of the shape, polarization and wavelength on the transmissivity and the diffraction angle distribution. The rigorous coupled-wave analysis (RCWA) and the nonstandard finite-difference time-domain (NS-FDTD) algorithm were used for the calculation. We found that the triangular grating in TE mode had the least wavelength dependence in the resonance domain, and this was explained by two independent parameters - (depth/wavelength) and (diffraction order $\times$ wavelength / period), whose ratio of the contribution to the diffraction efficiency equation was dependent on grating shape and polarization.
\end{abstract}

Keywords: grating shape, resonance domain, diffraction distribution, wavelength dependence, color rendering, FDTD, RCWA

\section{Introduction}

Scalar approximations such as those used in Snell's law and the Sweat model [1] are simple and intuitive calculation methods. These approximations can be applied to gratings of various shapes when the diffraction effect is not pronounced or when evaluation of the diffraction efficiency is not important. If the grating comprises an array of slits, the Fraunhofer diffraction approximation [2] can be applied, and the diffraction effect can be considered when determining the diffraction efficiency from the slit period and slit width.

In this decade, the scattering [3], antireflection [4-6], polarization selection [7, 8], and focusing characteristics [9, 10] of gratings in the resonance domain have attracted considerable interest, as these gratings have potential applications in displays, LEDs, solar panels, image pickup devices, etc. The period of a grating in the resonance domain ranges from one-half of the wavelength of the incident light to several dozens of the wavelength, and further, such a grating shows remarkable diffraction effects. The dependence of the angular transmissivity spectrum (transmissivity plotted as a function of the diffraction angle) on the grating period and grating depth has been discussed in several studies [11-15].

Several studies have also investigated the effect of the shape of the grating on the angular transmissivity spectrum. Yokomori calculated the first-order diffraction efficiencies of gratings having different shapes and *Corresponding author

Email address: hoshino@gabor.bk.tsukuba.ac.jp (Tetsuya Hoshino) 
periods/wavelengths less than 3 [16]. Suratteau et al. calculated the transmissivity of conducting wire gratings having a variety of shapes and periods less than the wavelength of the incident light [17].

Lately, studies on the wavelength dependence of angular transmissivity spectra have gained interest because the antireflective effect shown by a grating can help improve the energy efficiency of the LEDs in which it is used [18-21]. Gratings in the resonance domain can act as excellent antireflective structures [4]; however, such gratings sometimes cause wavelength dispersion, as has been observed when using them in spectrometers. Color rendering plays an important role in white-light illumination. Wavelength dispersion leads to poor color rendering and thus affects the illumination quality $[22,23]$.

The aim of this study is to analyze the diffraction efficiencies of gratings in the resonance domain and use the results to design a grating with low wavelength dispersion. Light emitted from white LEDs is unpolarized and travels in a straight line. We also investigate the effect of grating shape and polarization on wavelength dispersion for the case where the incident light travels along a straight line, by using a physical model and an equation. To clarify this wavelength dependence, we compare a triangular grating with a rectangular grating and a sinusoidal grating.

\section{Calculation method}

Fig. 1a and $\mathrm{b}$ respectively show the cross sectional profiles of the triangular and rectangular two-dimensional gratings used in this study. The gratings are made of transparent materials that do not absorb light. Light of wavelength $\lambda$ is incident from air on surface corrugations with a period $\Lambda$, a depth $d$, and a refractive index $n(=1.5)$. In case A, light impinges on the grating from the air (Fig. 1a), whereas in case B, light impinges on the grating from the opposite direction (i.e., upwards from the substrate). The substrate thickness $(d s)$ is infinite.

Fig. 1. Grating profiles and the definitions of $\Lambda, d$, and $d s$ : (a) triangular grating and (b) rectangular grating. Fill factor $=0.5$ and aspect ratio $=1 ; \theta^{\prime}$ is the incidence angle.

We used the nonstandard finite-difference time-domain (NS-FDTD) algorithm [24,25] to compute the scattered fields generated by an infinite plane wave incident on the gratings. In addition, we used a new nonstandard (NS) [26] version of the Mur absorbing boundary condition $(\mathrm{ABC})$, which gives a higher absorption than the standard Mur $\mathrm{ABC}$ but has the same low computational cost. We computed the scattered fields generated by the two dimensional surface relief gratings shown in Fig. 1. $d s$ is modelled as infinite by imposing an $\mathrm{ABC}$ at the base (see Fig. 1), while infinite periodicity was modelled by imposing periodic boundary conditions on the left and right (see Fig. 2).

We performed the same calculations using rigorous coupled-wave analysis (RCWA) [27-29] and computed the transmission spectra as functions of the incidence angle, polarization and wavelength. The periodic grating gives diffraction pattern of many diffraction orders and RCWA calculates diffraction efficiency of every diffraction order. 
These RCWA calculations were performed using DiffractMOD ${ }^{\mathrm{TM}} 1.5$ (RSoft Design Group, Ossining, NY, USA).

\section{Diffraction efficiency equations}

When wavelength changes for an infinite periodic grating, $\lambda / \Lambda<<1$ and visible wavelengths, the transmissive diffraction efficiency for the diffraction order $i$ of TE and TM modes are respectively [12] (see the Appendix):

$$
\begin{gathered}
T_{T E}(i) \approx E(i \lambda / \Lambda, d / \lambda), \\
T_{T M}(i) \approx M(i \lambda / \Lambda, \lambda / \Lambda, d / \lambda),
\end{gathered}
$$

where $E$ is a function of two independent parameters and $M$ is a function of three independent parameters.

According to Eq. (1), $T_{T E}(i)$ can be expressed by parameters $i \lambda / \Lambda$ and $d / \lambda$, whereas $T_{T M}(i)$ requires $\lambda / \Lambda$ (which is not multiplied by $i$ ) in addition to these two terms.

Envelope of angular transmissivity spectrum has peaks. The peak can have maximum diffraction efficiency $i_{\max }$. When $\lambda / \Lambda$ is small enough, wavelength dispersion becomes small and $T_{T E}\left(i_{\max }\right)$ should be constant against wavelength. In that case $i_{\max } \lambda / \Lambda$ is constant and the effect of $d / \lambda$ is negligible [12].

The angular transmissivity spectrum can be wavelength independent, if the envelope of angular transmissivity spectrum is wavelength independent as shown in the previous paper for TE mode [12]. In that case, as wavelength increases $i$ decreases and $i \lambda / \Lambda$ becomes constant against wavelength. As a result, the envelope does not change against wavelength very much.

\section{Electric field and angular distribution results}

We hypothesize that $i \lambda / \Lambda$ is the dominant parameter in the transmission function for the triangular grating in TE mode because there is likely to be little interference between waves reflected from the apex and bottom of the groove. On the other hand, the interference between waves reflected from the top and bottom of the rectangular grating may be large, so that the transmission is strongly affected by both $i \lambda / \Lambda$ and $d / \lambda$.

Figs. 2 and 3 show the electric fields calculated for the gratings with $\Lambda / \lambda=2$ and an aspect ratio of unity for case A. The incident light is TE polarized. As to relative phase and intensity, the maximum value of the absolute value is normalized by 3 or 6 . The relative phase shown in Fig. 2 is the real part of the electric field, whereas the relative intensity shown in Fig. 3 is the electric field strength. These results corroborate the above-mentioned hypothesis. These figures show that the electric field $(\boldsymbol{E})$ is distributed in equidistance along the direction of incident light in rectangular grating and it seems like interference along $z$ axis, whereas such an interference fringe is not seen in triangular grating. By comparison, in case B, the electric field distribution of the triangular grating in TM mode has 
more stripes along the $x$ axis than that of TE mode; thus the electric field distribution of TM mode is similar to that of the rectangular grating.

Fig. 2. (a) Coordinate system for the FDTD calculation and (b) Relative phase values. (c) The phase distribution of scattered light in the TE ( $\boldsymbol{E}$ in the $x$ - $z$ plane) mode for the triangular grating with a period of $2 \lambda$ and aspect ratio of 1 in case A. (d) Phase distribution of the scattered light in the TE mode for the rectangular grating with a period of $2 \lambda$ and aspect ratio of 1 in case $A$.

Fig. 3. (a) Relative intensity values. (b) The intensity distribution of scattered light in the TE mode for the triangular grating with a period of $2 \lambda$ and aspect ratio of 1 in case A. (c) The intensity distribution of the scattered light in the TE mode for the rectangular grating with a period of $2 \lambda$ and aspect ratio of 1 in case $\mathrm{A}$.

When $T_{T E}(i)$ has constant $i \lambda / \Lambda$ and $\lambda$ varies, the total reflectivity (i.e., the sum of all the diffraction efficiencies) will vary periodically with $\Lambda / \lambda$ and the angular distribution will be constant in the resonance region [12]. The reflectivities of the triangular and rectangular gratings were computed using the RCWA; the results are respectively shown in Figs. 4 and 5 (for aspects ratios of 0.5 and 1 and of 1 and 2, respectively). As the aspect ratio is constant along the horizontal axis $(\Lambda / \lambda)$ in Fig. 4, this figure also shows the $d / \lambda$ dependences of the reflectivity of the triangular grating, allowing the results in Fig. 4 to be directly compared with those in Fig. 5. The reflectivity of the triangular grating is periodic with the same period as the grating, whereas the reflectivity of the rectangular grating varies periodically with the groove depth. Then, for example, when Fig. 4 is redrawn against $d / \lambda$, the first fluctuation around $\Lambda / \lambda=2$ shifts by the aspect ratio. Thus, the wavelength dependence of the reflectivity depends on the grating shape. When $\Lambda / \lambda$ is close to 2 , the reflectivity clearly varies periodically with the wavelength and the electric fields of triangular and rectangular gratings differ significantly (see Figs. 2 and 3). As the gratings have no absorption, the total transmission can be found by subtracting the total reflection from unity, which is helpful when we consider the angular transmission spectrum in the following section.

Fig. 4. Wavelength dependence of the reflectivity of the triangular grating in case A. The polarization modes are TE and TM. The aspect ratios are 0.5 and $1 . d s$ is set to infinity.

Fig. 5. Wavelength dependence of the reflectivity of the rectangular grating in case A. The polarization modes are TE and TM. The aspect ratios are 1 and 2. $d s$ is set to infinity. 
The gratings in Fig. 1 were used to calculate the results shown in Figs. 6 to 9. We used grating periods $(\Lambda / \lambda)$ of 9.1 and 22.7. Even when $\Lambda / \lambda$ differs as much as $\pm 20 \%$ from 9.1 , the envelope of the angular spectrum of the triangular grating does not change very much [12]. There is little diffraction when $\Lambda / \lambda$ is 22.7 . If the angles of the maximum diffraction efficiencies for $\Lambda / \lambda 9.1$ and 22.7 are almost the same, it turns out that the angle of the maximum diffraction efficiency does not change as $\Lambda / \lambda$ varies from 9.1 to 22.7 .

Fig. 6. Angular transmissivity spectra of the triangular grating as a function of the wavelength and incidence angle in case A. Graph legend: light incidence angle, mode of polarization, value of $\Lambda / \lambda$, respectively.

Fig. 7. Angular transmissivity spectra of the rectangular grating as a function of the wavelength and incidence angle in case A. Graph legend: light incidence angle, mode of polarization, value of $\Lambda / \lambda$, respectively.

Fig. 8. Angular transmissivity spectra of the sinusoidal grating as a function of the wavelength and incidence angle in case A. Graph legend: light incidence angle, mode of polarization, value of $\Lambda / \lambda$, respectively.

Fig. 9. Angular transmissivity spectra of the triangular grating for TM mode as a function of the wavelength and incidence angle in case A. Graph legend: light incidence angle, mode of polarization, value of $\Lambda / \lambda$, respectively.

The aspect ratio and refractive index do not change with wavelength in calculation. By performing calculations (results not shown), we found that the variation in the refractive index of common transparent materials with wavelength does not greatly affect the angular transmission spectrum of the triangular grating in the visible wavelength region. Consequently, we neglected the change in the refractive index with wavelength. Figs. 6 and 7 respectively show the transmission spectra of the triangular and rectangular gratings in TE mode. When the incidence angle $\left(\theta^{\prime}\right)$ is in the range $0^{\circ} \leq \theta^{\prime} \leq 10^{\circ}$, the rectangular grating has a large wavelength dispersion, whereas the triangular grating has a low wavelength dispersion. Fig. 8 shows the angular transmission spectrum of a sinusoidal grating; it also has a large wavelength dispersion compared with that of a triangular grating. More diffraction results for the triangular grating were given in previous studies [12,13].

Table 1. $\Delta \theta_{\text {average }}$ of the three shapes for different polarization and direction of incident light.

We consider that the variation in the angle of the peak in the angular spectrum with wavelength reflects the wavelength dependence and it can be used as an index for the wavelength dependence. We introduce a new 
parameter, $\Delta \theta$, which is defined as the difference between the angles at which the maximum diffraction efficiencies occur for $\Lambda / \lambda=9.1$ and 22.7. $\Delta \theta_{\text {average }}$ is the average of the values $\Delta \theta$ for incidence angles of $0^{\circ}, 5^{\circ}, 10^{\circ}, 15^{\circ}, 20^{\circ}$ and $30^{\circ}$. These incidence angles are selected to be much less than the critical angle of the total reflection, which is determined by the refractive indices for regions 1 and 3. Table 1 shows the calculated values of $\Delta \theta_{\text {average }}$ for triangular, rectangular and sinusoidal gratings. For reference, the result of right triangle shape is added to the table. One of the base angles is $90^{\circ}$ in Fig. 1 . We calculated right triangle with $d / \Lambda=1$, and checked the effect of asymmetry shape. Symmetric triangle is abbreviated by "S-triangle", and asymmetric triangle is abbreviated by "A-triangle". The diffraction efficiency is dependent on whether the sign of incident angle $\theta$ is minus or plus, as the shape is asymmetric. When incident light has same slant with asymmetric triangle, it is denoted by "A-Triangle-s". Otherwise, when it has opposite slant, it is denoted by "A-Triangle-o". In this table, TE-A and TE-B indicate the TE mode and incident light direction for cases $\mathrm{A}$ and $\mathrm{B}$, respectively. We also calculated $\Delta \theta_{\text {average }}$ for the TM mode. In case A, $\Delta \theta_{\text {average }}$ of the TM mode for the triangular grating is exactly the same as that for the TE mode (see Figs. 6 and 9). On the other hand, in case $\mathrm{B}, \Delta \theta_{\text {average }}$ of the TM mode is greater than $\Delta \theta_{\text {average }}$ of the TE mode, but it is still less than that of the rectangular grating.

Two effects are expected for the right triangle shape. One is the asymmetric shape effect. The other is the angle effect between incident light and the slant of the triangle. Here we compare "A-Triangle-s" with "A-Triangle-o". As the angle between the slant and incident light becomes larger, the wavelength dependency becomes larger. When we compare the asymmetric effect with the angle effect, the former seems to be less remarkable than the latter in case A, because the difference of symmetric and asymmetric effects on $\Delta \theta_{\text {average }}$ is small besides the angle effect. On the other hand, in case B, the asymmetric effect is remarkable. As to polarization, TE and TM modes of the asymmetric triangle have similar wavelength dependency, like those of the symmetric triangle.

\section{Discussion}

The diffraction angle distribution is independent of the wavelength in the resonance domain, if it is dominated by $i \lambda / \Lambda$ [12]. This suggests that the diffraction efficiency is dominated only by $i \lambda / \Lambda$ in Figs. 6 and 9.

First, we consider the relationship between the diffraction distribution and the equations. Although Eqs. (1) and (2) differ somewhat, for both TE and TM modes, the wavelength dispersion of the triangular grating is smaller than that of the rectangular grating for case A. Moreover, for case B, the wavelength dispersion of the triangular grating for the TM mode is still less than that of the rectangular grating, although the wavelength dispersion for case B is larger than that for case $\mathrm{A}$ of the triangular grating.

When the parameters are limited to $i, \lambda, \Lambda$ and $d$, the only independent parameters that is not common to both the 
TE and TM modes is $\lambda / \Lambda$. For the triangular grating, the main parameter seems to be $i \lambda / \Lambda$ for a change in $\lambda$ and the effect of $\lambda / \Lambda$ is small for case A for the TM mode. On the other hand, for case B, although the effect of $\lambda / \Lambda$ is not remarkable, the wavelength dispersion is larger than that of case A. The difference between the TE and TM modes may be $\lambda / \Lambda$ or the combination of $\lambda / \Lambda$ and $d / \lambda$. The direction of the light may also affect the angular spectrum, as the sign of $\lambda / \Lambda$ is opposite for cases A and B in Eq. (A.3) for the TM mode.

We next consider the relationship between the electric field and the equations to obtain a physical model. The TE mode equations reveal that $i \lambda / \Lambda$ and $d / \lambda$ are significant. If $d / \lambda$ is more important than $i \lambda / \Lambda$ as a parameter for the angular spectrum, the electromagnetic field along the $x$ axis may interfere. The FDTD algorithm results show that $i \lambda / \Lambda$ is a more important parameter for the triangular grating than for the rectangular grating. When the parameter $i \lambda / \Lambda$ determines the transmission spectra, the wavelength dependence is small [12]. Polarization can also affect the wavelength dependence, as the electric field distributions of the TE and TM modes along the $x$ axis are different for case B. The wavelength dependences of the rectangular and triangular gratings should be compared for the same direction of the incident light and for the same polarization. The triangular grating exhibits a smaller wavelength dependence than the rectangular grating.

Because the dominant parameter for the triangular grating is $i \lambda / \Lambda$ in the resonance domain, its angular spectrum varies little with wavelength. Our results demonstrate that a triangular grating is superior to rectangular and sinusoidal gratings when it is desired to suppress the wavelength dispersion of the angular spectrum.

\section{Conclusions}

To improve color rendering properties of diffracted white light, we have clarified the shape dependence of the transmissivity and its angular spectra using the NS-FDTD algorithm and the RCWA for the gratings in the resonance domain. We find that the triangular grating shows considerably less wavelength dispersion as compared to both the rectangular one and sinusoidal one, and that the triangular one has less wavelength dispersion in TE mode than in TM mode. Though the main parameter of triangular grating in TE mode is only $i \lambda / \Lambda$, other shapes or polarization has additional parameters $d / \lambda$ and/or $\lambda / \Lambda$, which cause wavelength dispersion.

\section{Appendix A. Diffraction efficiency in the TM mode}

To determine the diffraction efficiency as a function of depth or period, it is necessary to derive the equation for the electromagnetic field. In a previous paper, we demonstrated that when wavelength changed in the resonance domain, the diffraction efficiency in the TE mode had two independent parameters: $i \lambda / \Lambda$ and $d / \lambda$ [12]. In this appendix, we determine the independent parameters for the TM mode. For TE mode, the RCWA procedure for general dielectric surface-relief grating has been described in detail by Moharam and Gaylord [28]. We studied the 
equation for the TM mode to determine the independent parameters.

In Fig. 1, regions 1 and 3 are homogeneous dielectrics with relative permittivity of $\varepsilon_{\mathrm{I}}$ and $\varepsilon_{\text {III }}$, respectively. Region 2 consists of a periodic distribution of both dielectrics. To calculate the electromagnetic field of a periodic surface-relief grating, the grating in region 2 is sliced into $N$ layers along the $z$ axis (normal to the boundary of the regions) as shown by Moharam and Gaylord [28]. Each layer is represented by a Fourier expansion of the dielectric distribution along the $x$ axis. We start with the Fourier expansion of the $n$th layer.

The relative permittivity of the $n$th slab grating is expanded in a Fourier series as [28]

$$
\varepsilon_{n}\left(x, z_{n}\right)=\varepsilon_{\mathrm{I}}+\left(\varepsilon_{\mathrm{III}}-\varepsilon_{\mathrm{I}}\right) \sum_{h=-\infty}^{\infty}\left[\tilde{\varepsilon}_{h, n} \exp (j h K x)\right],
$$

where $z_{n}$ is the $z$ coordinate of the $n$th slab, $j$ represents $(-1)^{1 / 2}, K$ is the magnitude of the grating vector and is equal to $2 \pi / \Lambda$, and $\tilde{\varepsilon}_{h, n}$ is the normalized complex harmonic amplitude coefficient given by:

$$
\tilde{\varepsilon}_{h, n}=(1 / \Lambda) \int_{0}^{\Lambda} f\left(x, z_{n}\right) \exp (-j h K x) \mathrm{d} x,
$$

where $f\left(x, z_{n}\right)$ equals either zero or unity for the grating in Fig. 1[28].

Maxwell's theory gives two equations: one for the magnetic field vector and another for the electric field vector $[27,28]$. The electric and magnetic fields may be expressed as Fourier expansions in terms of the space-harmonic field; substituting the Fourier expansions into the above two equations results in second-order coupled-wave equations in terms of $U_{i, n}(z)[27,28]$, which is the $i$ th space-harmonic-normalized amplitude.

$$
\begin{aligned}
& \lambda^{2} \frac{\mathrm{d}^{2} U_{i, n}(z)}{\mathrm{d} z^{2}}-4 \pi j \lambda\left(\varepsilon_{0, n}-\varepsilon_{\mathrm{I}} \sin ^{2} \theta^{\prime}\right)^{1 / 2} \frac{\mathrm{d} U_{i, n}(z)}{\mathrm{d} z} \\
& +(2 \pi \lambda / \Lambda)^{2} i(m-i) U_{i, n}(z) \\
& +4 \pi^{2}\left(\varepsilon_{\mathrm{III}}-\varepsilon_{\mathrm{I}}\right) \sum_{h=1}^{\infty}\left[\tilde{\varepsilon}_{h, n} U_{i-h, n}(z)+\tilde{\varepsilon}_{h, n}^{*} U_{i+h, n}(z)\right] \\
& +(2 \pi \lambda / \Lambda)^{2} \sum_{h=-\infty}^{\infty}\left[\left(i-h-\frac{m}{2}\right) A_{h, n} U_{i-h, n}(z)=0 .\right.
\end{aligned}
$$

Here, $i$ is the space harmonic index, $\quad \tilde{\varepsilon}_{h, n}^{*}$ is the complex conjugate of $\tilde{\varepsilon}_{h, n}, \varepsilon_{0, n}$ is the average relative permittivity for the $n$th slab grating, and $A_{h, n}$ is given by:

$$
\frac{\left(\varepsilon_{\mathrm{III}}-\varepsilon_{\mathrm{I}}\right)}{\varepsilon_{\mathrm{n}}\left(\mathrm{x}, \mathrm{z}_{\mathrm{n}}\right)} \sum_{h=-\infty}^{\infty}\left[\tilde{\varepsilon}_{h, n} h \exp (j h K x)\right]=\sum_{h=-\infty}^{\infty}\left[A_{h, n} \exp (j h K x)\right]
$$

The parameter $m$ is given by: 


$$
m=\frac{2 \Lambda \varepsilon_{\mathrm{I}}^{1 / 2} \sin \theta^{\prime}}{\lambda} .
$$

Let us assume that the variable parameters are $i, \lambda, \Lambda$ and $d$. When $z$ is replaced by $z / \lambda$ in the equations, all the coefficients on the left-hand side of Eq. (A.3) can be expressed in terms of $i \lambda / \Lambda, \lambda / \Lambda$ and $d / \lambda$ [12]. Parameter $\lambda / \Lambda$ comes from the fifth term in Eq. (A.3).

The transmissivity is derived from Eq. (A.3). Eq. (A.3) is solved as a matrix equation whose state spaces are a linear combination of $U_{i, n}(z)$ and $\mathrm{d} U_{i, n}(z) / \mathrm{d} z$ [28]. The equation has boundary conditions for the $N$ layers [27-29] and the boundary condition is obtained for the electric and magnetic fields for each layer. For example, the boundary condition between the $N$ th layer and region 3 is given by:

$$
T_{i}=\sum_{q^{\prime}=1}^{2 s} C_{q^{\prime}, N} \omega_{p^{\prime}, q^{\prime}, N} \exp \left\{\left[\lambda \lambda_{q^{\prime}, N}-j \lambda\left(\sigma_{i, N} \hat{z}\right)\right] d / \lambda\right\}
$$

for the tangential magnetic field and

$$
-j\left(\mathbf{k}_{3 i} \hat{z} / n_{\mathrm{III}}^{2}\right) T_{i}=\sum_{q^{\prime}=1}^{2 s} C_{q^{\prime}, N} \omega_{p^{\prime}, q^{\prime}, N}\left[\lambda_{q^{\prime}, N}-j\left(\boldsymbol{\sigma}_{i, N} \hat{z}\right)\right] \exp \left\{\left[\lambda \lambda_{q^{\prime}, N}-j \lambda\left(\boldsymbol{\sigma}_{i, N} \hat{z}\right)\right] d / \lambda\right\},
$$

for the tangential electric field, where $n_{\mathrm{III}}$ is the refractive index in region $3, T_{i}$ is the normalized amplitude of the $i$ th transmitted wave into region $3, \lambda_{q^{\prime}, N}$ and $\omega_{p^{\prime}, q^{\prime} N}$ are respectively the eigenvalues and eigenvectors of the matrix of the state space. $p$ ' and $q$ ' are respectively the row and column indices of the eigenvector matrix, $s$ is equal to the number of diffracted orders retained in the analysis, $C_{q^{\prime}, N}$ are unknown constants to be determined by the boundary conditions, and $\hat{z}$ is a unit vector along the $z$ axis. $p$ ' is chosen to correspond to the $i$ th diffraction wave. $\boldsymbol{\sigma}_{i, N} \hat{z}$ is given by:

$$
\boldsymbol{\sigma}_{i, n} \hat{z}=\frac{2 \pi}{\lambda} \sqrt{\varepsilon_{0, n}-\varepsilon_{\mathrm{I}} \sin ^{2} \theta^{\prime}}
$$

$\mathbf{k}_{3, i} \hat{z}$ is given by the later Eq. (A.11).

The boundary condition between the first layer and the region 1 is similar to that of TE mode and the difference of the equation is only coefficient $\left(1 / n_{\mathrm{I}}\right)^{2}$ like the coefficient of Eq. (A.7) is $\left(1 / n_{\mathrm{III}}\right)^{2}$. Moreover, the boundary condition between $n$th and $n+1$ th slab gratings is formally same to that of TE mode [28].

$T_{i}$ is solved by the differential equation and these boundary conditions. All equation and conditions can be written by parameters $i \lambda / \Lambda, \lambda / \Lambda$ and $d / \lambda$. The diffraction efficiency is given in terms of $T_{i}$ [29] as

$$
D E_{3 i}=\operatorname{Re}\left[\left(n_{\mathrm{I}}^{2} \mathbf{k}_{3 i} \hat{z}\right) /\left(n_{\mathrm{III}}^{2} \mathbf{k}_{10} \hat{z}\right)\right] T_{i} T_{i}^{*}
$$

where $D E_{3 i}$ is $i$ th diffraction efficiency of transmission and is denoted by $T_{T M}(i)$ in the main text. $\mathbf{k}_{10}$ and $\mathbf{k}_{3 i}$ are wave vectors in regions 1 and 3 respectively, and their $z$ components are given by:

$$
\mathbf{k}_{10} \hat{z}=\frac{2 \pi}{\lambda} \cos \theta^{\prime} \sqrt{\varepsilon_{\mathrm{I}}},
$$


and

$$
\mathbf{k}_{3 i} \hat{z}=\frac{2 \pi}{\lambda} \sqrt{\varepsilon_{\mathrm{III}}-\left[\left(\varepsilon_{\mathrm{III}}\right)^{1 / 2} \sin \theta^{\prime}-i \lambda / \Lambda\right]^{2}} .
$$

We consider $i, \lambda, \Lambda$ and $d$ as the parameters to be set. The solution of the diffraction efficiency equation for the grating is given by the total coefficient matrix of Eq. (A.3), whose eigenvalues, eigenvectors, and coefficients are dominated by $i \lambda / \Lambda, \lambda / \Lambda$ and $d / \lambda$, which can be easily derived [12]. $\lambda / \Lambda$ is additional parameter when the parameters of TM mode are compared with those of TE mode.

Besides of the parameters $i \lambda / \Lambda, \lambda / \Lambda$ and $d / \lambda$, s changes as wavelength changes in Eq. (A.6). $s$ is roughly proportional to $\Lambda / \lambda[2]$, but is not parameter of each terms of equations. For example, $2 s$ is the number of exponential terms in Eq. (A.6) or (A.7). For a triangular grating in the resonance domain, the incident light is bended according to Snell's laws and the each ray is broadened according to Fraunhofer theory in TE mode [30]. Then $\lambda / \Lambda$ and $d / \lambda$ may affect the angle of the peak as these parameters should affect the bending direction of the ray. $s, \lambda / \Lambda$ and $d / \lambda$ may affect the peak width of the envelope of the angular spectrum. Moreover, the effect of $s$ on the peak width against wavelength was found to be small in the visible range of light when $\lambda / \Lambda$ is less than 1/7 in TE mode [12]. If the effect of $s$ of TM mode on the peak is similar to that of TE mode, that may be small. As the calculated results of this paper suggest the wavelength dependence of the peak width of TM mode is as small as that of TE mode, this approximation is valid. As all parameters of $D E_{3 i}$ are expressed by $i \lambda / \Lambda, \lambda / \Lambda$ and $d / \lambda$, Eq. (2) is derived. Moreover, if the angular transmissivity spectrum of TM mode is wavelength independent, the effect of $\lambda / \Lambda$ and $d / \lambda$ also turns out to be small.

If $\lambda$ changes as small as $\pm 20 \%$, Eq. (1) can often be considered as function of only $i \lambda / \Lambda$. However, when $\lambda$ becomes less than half or more than double, the effect of $s$ and $d / \lambda$ is not negligible and the precise effect of them on the diffraction efficiency is a little difficult to predict [30]. The peak width of the envelope pattern of the diffraction sharpens [30] and the angle difference of each diffraction order narrows. The former brings about larger diffraction efficiency at the peak of angular spectrum. On the other hand, the latter brings about smaller averaged diffraction efficiency on the number of diffraction orders. The above wavelength dependency of Eq. (1) may also be applicable to Eq. (2).

Apart from the grating shape, the direction of incident light may be important factor for the wavelength dependency. As the wavelength dependence for cases A and B is different in this paper's results of TM mode, the relationship between cases $\mathrm{A}$ and $\mathrm{B}$ as to parameter $\lambda / \Lambda$ seems to depend on the sign of the fifth term of Eq. (A.3), which changes as the direction of light becomes opposite. $T_{T M}(i)$ of Eq. (2) is possibly affected by the sign.

\section{References}

[1] W. C. Sweatt, J. Opt. Soc. Am. 67 (1977) 803.

[2] M. Born, E.Wolf, Principles of Optics, : Electromagnetic Theory of Propagation, Interference and Diffraction 
of Light, 7th expanded ed., Cambridge University Press, Cambridge, 1999.

[3] Y. T. Lua, S. Chia, Opt. Commun. 214 (2002) 55.

[4] A. Fujimoto, K. Asakawa, Nano-structured surface fabrication for higher luminescent led by self-assembled block copolymer, in: 2005 International Microprocesses and Nanotechnology Conference, IEEE, Tokyo, Japan, 2005, pp. 76.

[5] A. Parretta, A. Sarno, P. Tortora, H. Yakubu, P. M. J. Zhao, A. Wang, Opt. Commun. 172 (1999) 139.

[6] S. Hava, M. Auslender, Solar Energy Materials and Solar Cells 61 (2000) 143.

[7] E. Noponen, A. Vasara, J. Turunen, J. M. Miller, M. R. Taghizadeh, J. Opt. Soc. Am. A 9 (1992) 1206.

[8] H. Haidner, D. Dias, L. L. Wang, T. Tschudi, Pure Appl. Opt. 7 (1998) 1347.

[9] Y. Wang, W. Yun, C. Jacobsen, Nature 424 (2003) 50.

[10] T. Nakai, H. Ogawa, Development of 3-layer diffractive optical elements employed for wide incident angles, in: Technical Digest of ICO’04 International Conference Optics and Photonics in technology Frontier Tokyo, Tokyo, 2004, pp. 547.

[11] M. A. Golub, A. A. Friesem, J. Opt. Soc. Am. A 22 (2005) 1115.

[12] T. Hoshino, M. Itoh, T. Yatagai, Appl. Opt. 46 (2007) 648.

[13] T. Hoshino, S. Banerjee, M. Itoh, T. Yatagai, Appl. Opt. 46 (2007)7948.

[14] M. A. Golub, A. A. Friesem, J. Opt. Soc. Am. A 24 (2007) 687.

[15] T.-X. Lee, K.-F. Gao, W.-T. Chien, C.-C. Sun, Opt. Express 15 (2007) 6670.

[16] J. Y. Suratteau, R. Petit, Int. J. Infrared Millimeter Waves 6 (1985) 831.

[17] K. Yokomori, Appl. Opt. 23 (1984) 2303.

[18] K. Ishihara, M. Fujita, I. Matsubara, T. Asano, S. Noda, H. Ohata, A. Hirasawa, H. Nakada, N. Shimoji, Appl. Phys. Lett. 90 (2007) 1.

[19] Y.-G. Ju, B.-W. Lee, Jpn. J. Appl. Phys. 46 (2007) 5153.

[20] H. Masui, S. Nakamura, S. P. DenBaars, Appl. Opt. 47 (2008) 88.

[21] S. Pimputkar, J. S. Speck, S. P. DenBaars, S. Nakamura, Nat. Photonics 3 (2009) 180.

[22] Y. Ohno, Proc. of SPIE 5530 (2004) 88.

[23] M. Funato, T. Kondou, K. Hayashi, S. Nishiura, M. Ueda, Y. Kawakami, Y. Narukawa, T. Mukai, Appl. Phys. Express 1 (2008) 1.

[24] J. B. Cole, S. Banerjee, M. Haftel, in: R. E. Mickens (Ed.), Advances in the applications of nonstandard finite difference schemes, World Scientific, Singapore, 2006, pp. 89.

[25] . B. Cole, S. Banerjee, J. Diff. Eqns. 9 (2003) 1099.

[26] J. B. Cole, S. Banerjee, Improved version of the second-order Mur absorbing boundary condition based on a nonstandard finite difference model, in: The 23rd Annual Review of Progress in Applied Computational Electromagnetics, Applied Computational Electromagnetics, Verona, Italy, 2007, pp. 1531. 
[27] M. G. Moharam, T. K. Gaylord, J. Opt. Soc. Am. A 73 (1983) 451.

[28] M. G. Moharam, T. K. Gaylord, J. Opt. Soc. Am. A 72 (1982) 1385.

[29] M. G. Moharam, D. A. Pommet, E. B. Grann, T. K. Gaylord, J. Opt. Soc. Am. A 12 (1995) 1077.

[30] T. Hoshino, S. Banerjee, M. Itoh, T. Yatagai, J. Opt. Soc. Am. A 26 (2009) 715. 


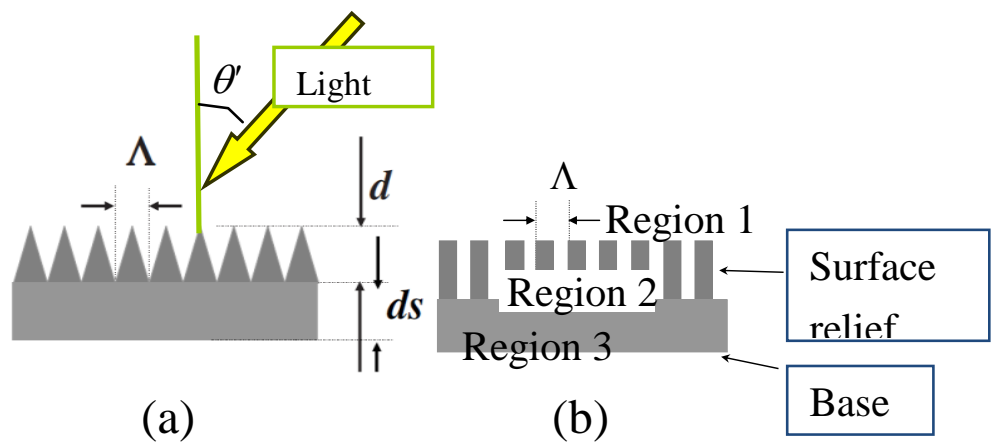

Fig. 1. Grating profiles and the definitions of $\Lambda, d$, and $d s$ : (a) triangular grating and (b) rectangular grating. Fill factor $=0.5$ and aspect ratio $=1 ; \theta^{\prime}$ is the incidence angle. 


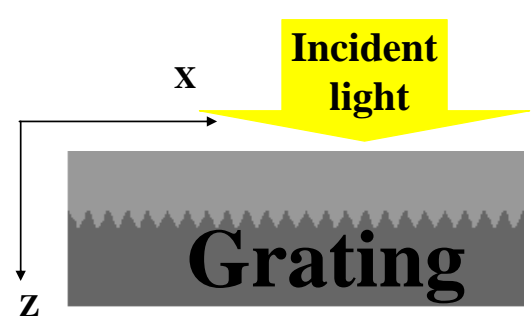

(a)

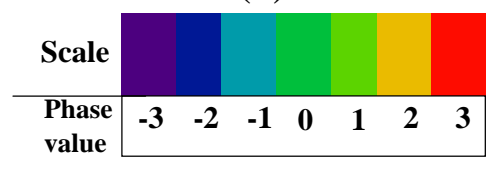

(b)

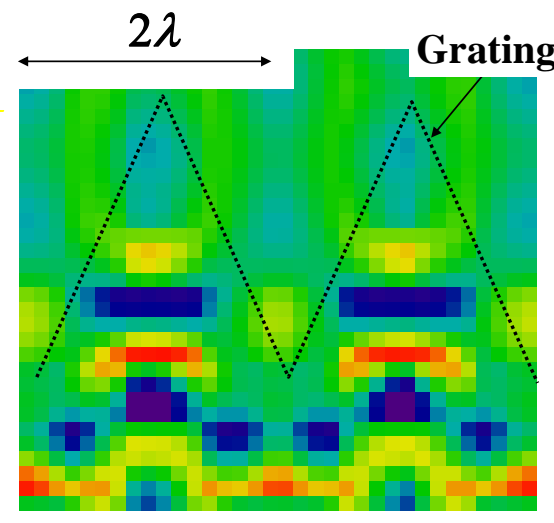

(c)

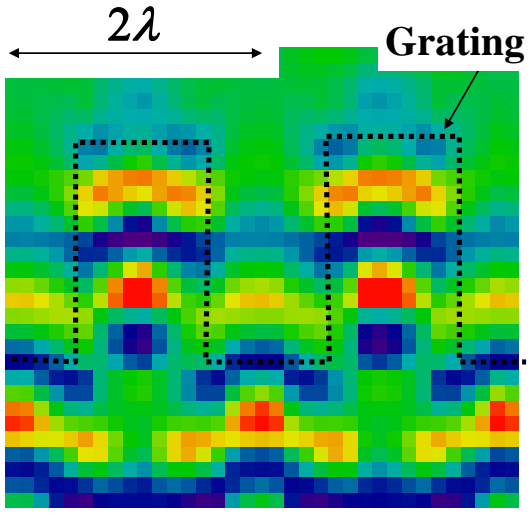

(d)

Fig. 2. (a) Coordinate system for the FDTD calculation and (b) Relative phase values. (c) The phase distribution of scattered light in the TE ( $\boldsymbol{E}$ in the $x-z$ plane) mode for the triangular grating with a period of $2 \lambda$ and aspect ratio of 1 in case A. (d) Phase distribution of the scattered light in the TE mode for the rectangular grating with a period of $2 \lambda$ and aspect ratio of 1 in case $A$. 

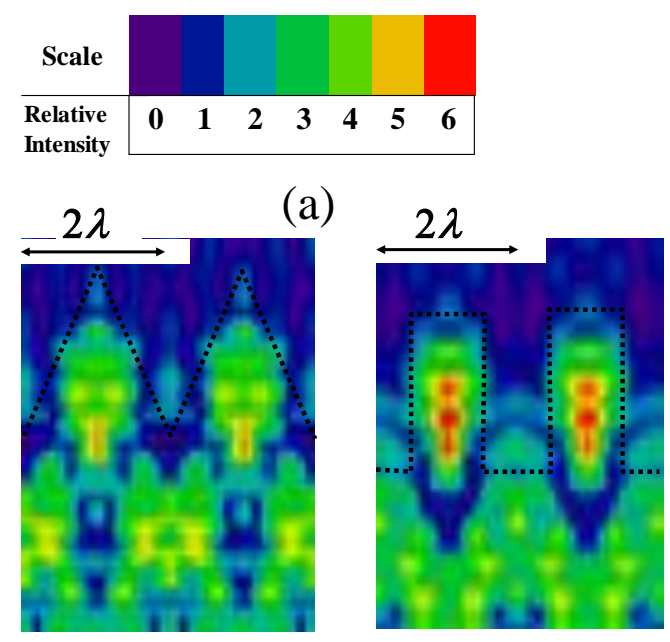

(b)

(c)

Fig. 3. (a) Relative intensity values. (b) The intensity distribution of scattered light in the TE mode for the triangular grating with a period of $2 \lambda$ and aspect ratio of 1 in case A. (c) The intensity distribution of the scattered light in the TE mode for the rectangular grating with a period of $2 \lambda$ and aspect ratio of 1 in case A. 


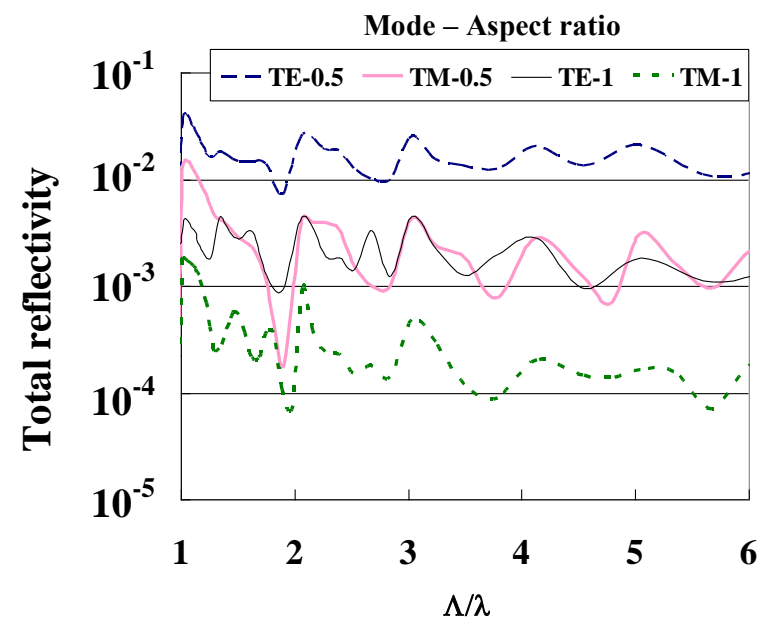

Fig. 4. Wavelength dependence of the reflectivity of the triangular grating in case A. The polarization modes are TE and TM. The aspect ratios are 0.5 and $1 . d s$ is set to infinity. 


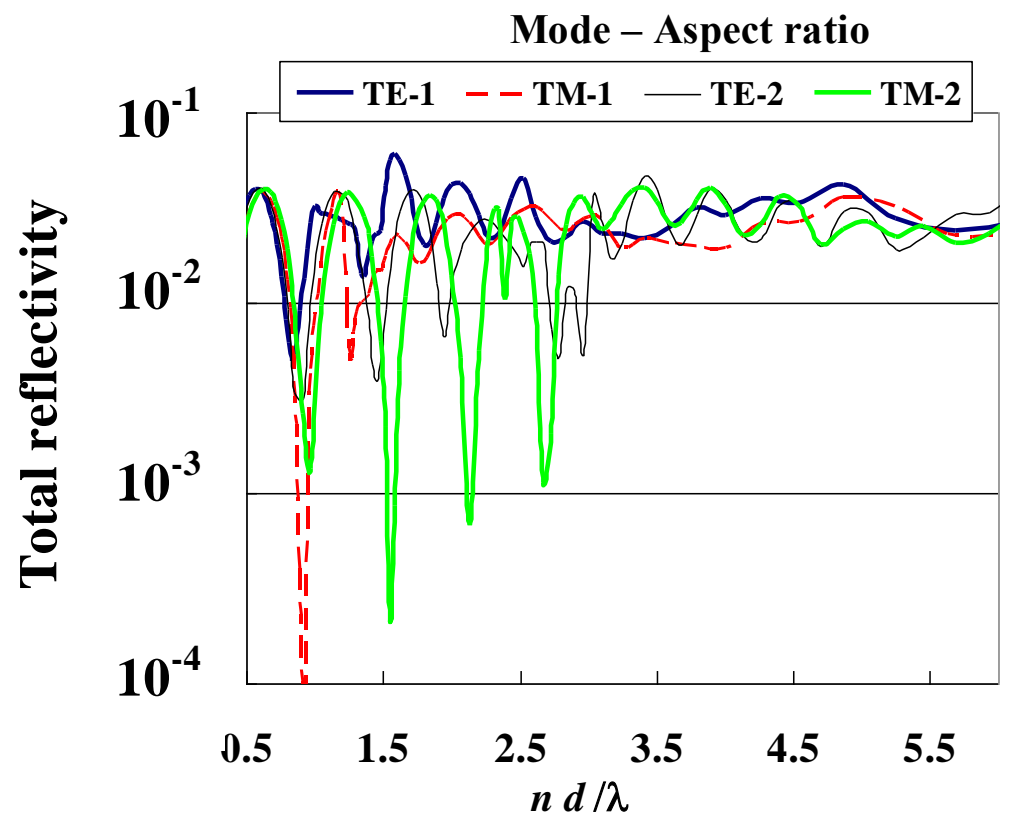

Fig. 5. Wavelength dependence of the reflectivity of the rectangular grating in case A. The polarization modes are TE and TM. The aspect ratios are 1 and $2 . d s$ is set to infinity. 

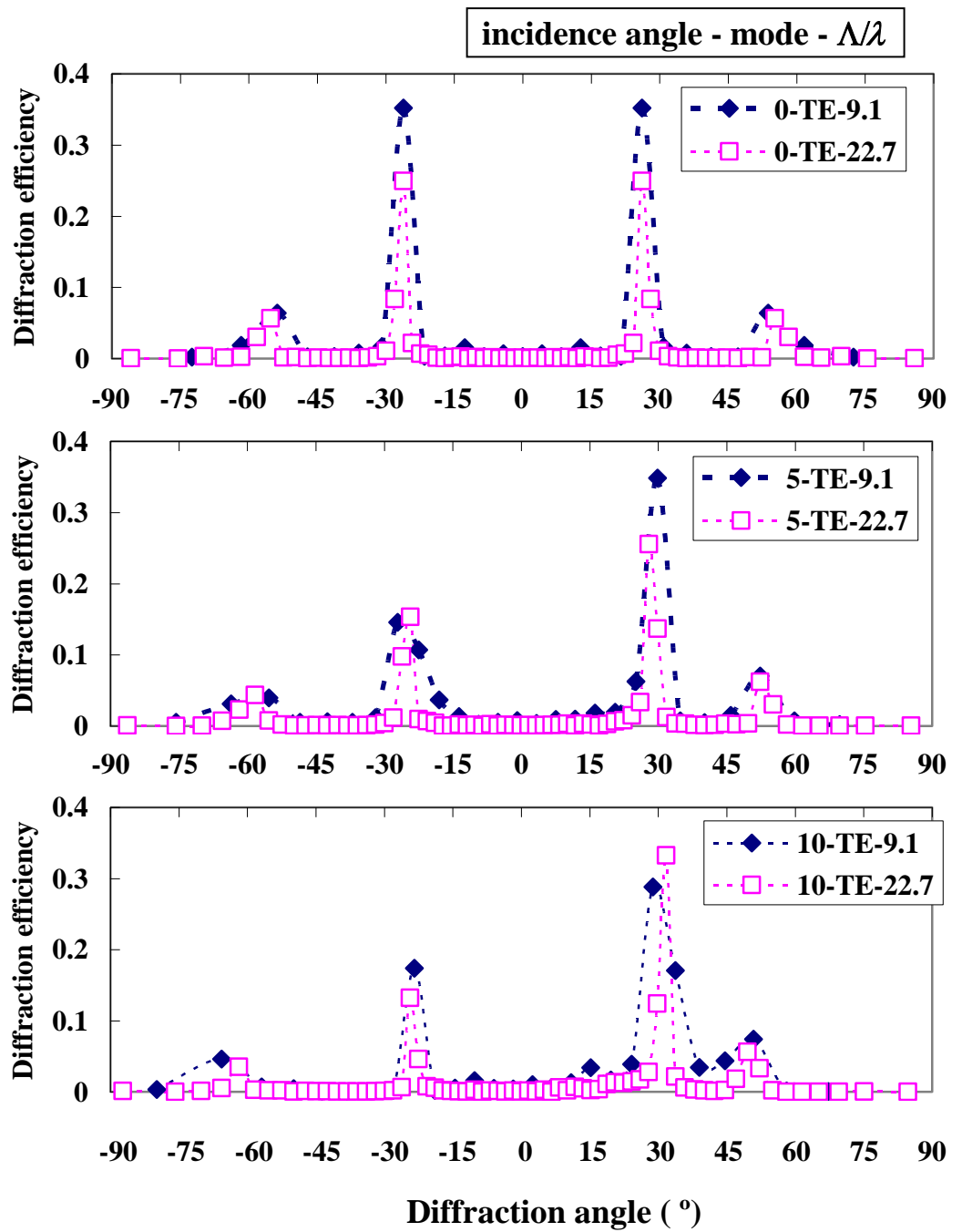

Fig. 6. Angular transmissivity spectra of the triangular grating as a function of the wavelength and incidence angle in case A. Graph legend: light incidence angle, mode of polarization, value of $\Lambda / \lambda$, respectively. 

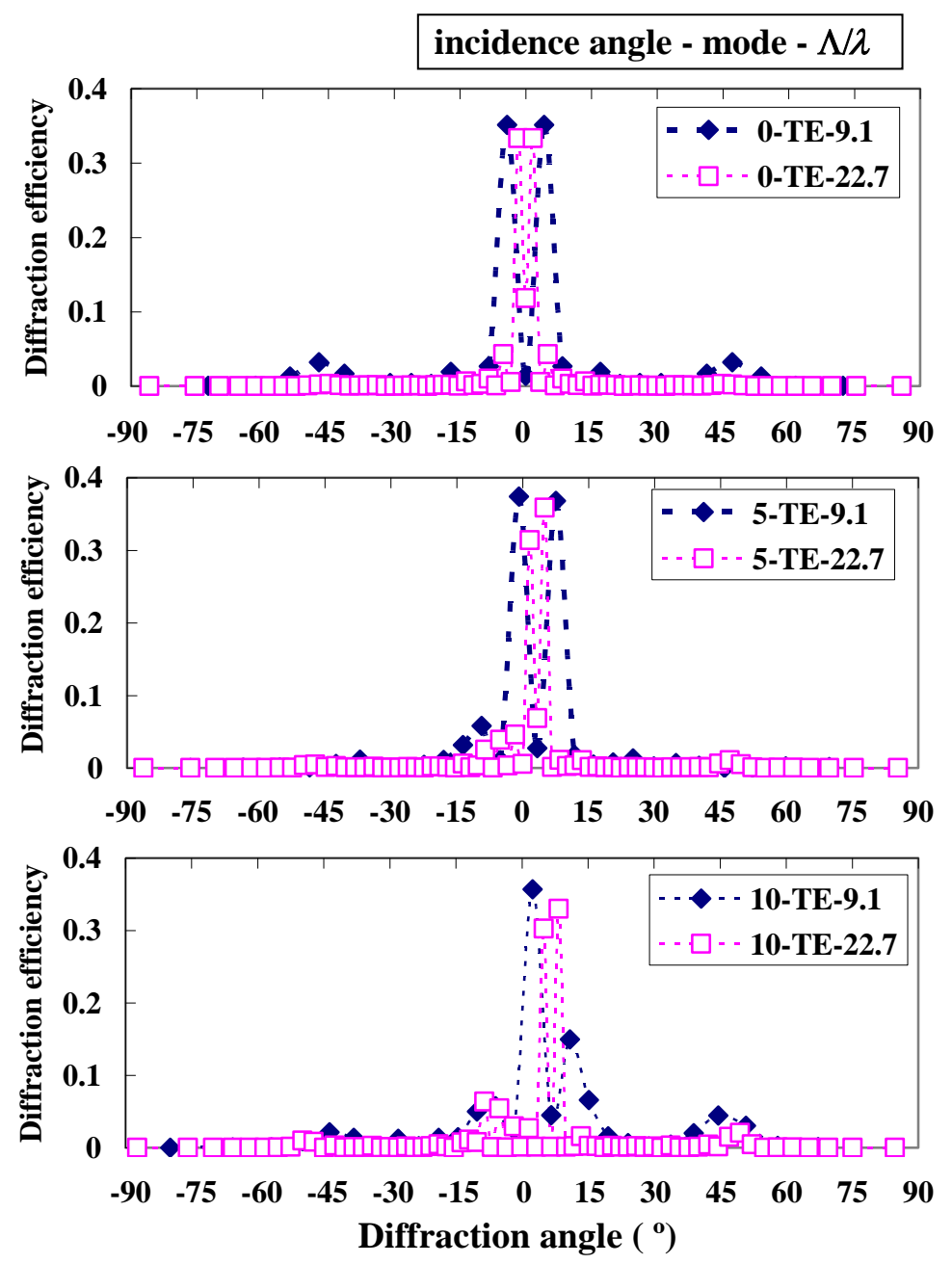

Fig. 7. Angular transmissivity spectra of the rectangular grating as a function of the wavelength and incidence angle in case A. Graph legend: light incidence angle, mode of polarization, value of $\Lambda / \lambda$, respectively. 

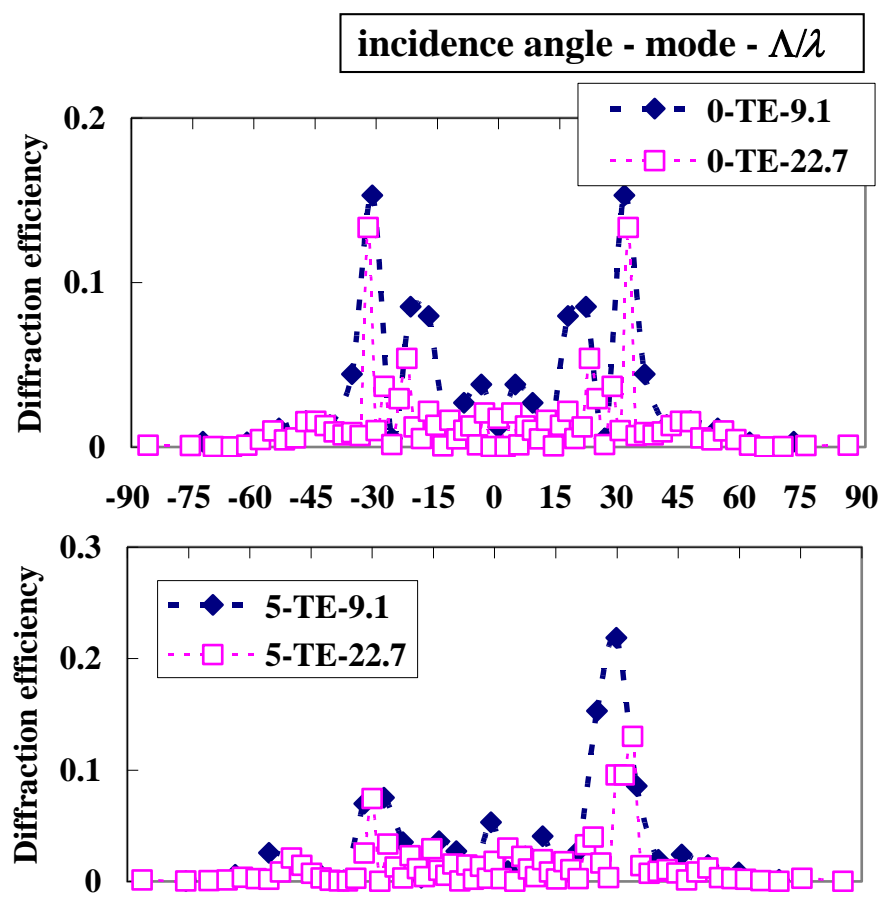

$\begin{array}{lllllllllllll}-90 & -75 & -60 & -45 & -30 & -15 & 0 & 15 & 30 & 45 & 60 & 75 & 90\end{array}$

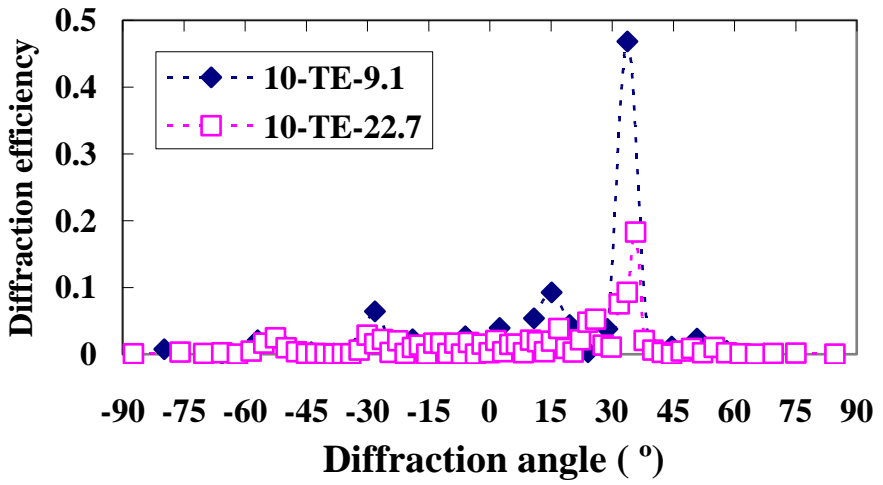

Fig. 8. Angular transmissivity spectra of the sinusoidal grating as a function of the wavelength and incidence angle in case A. Graph legend: light incidence angle, mode of polarization, value of $\Lambda / \lambda$, respectively. 

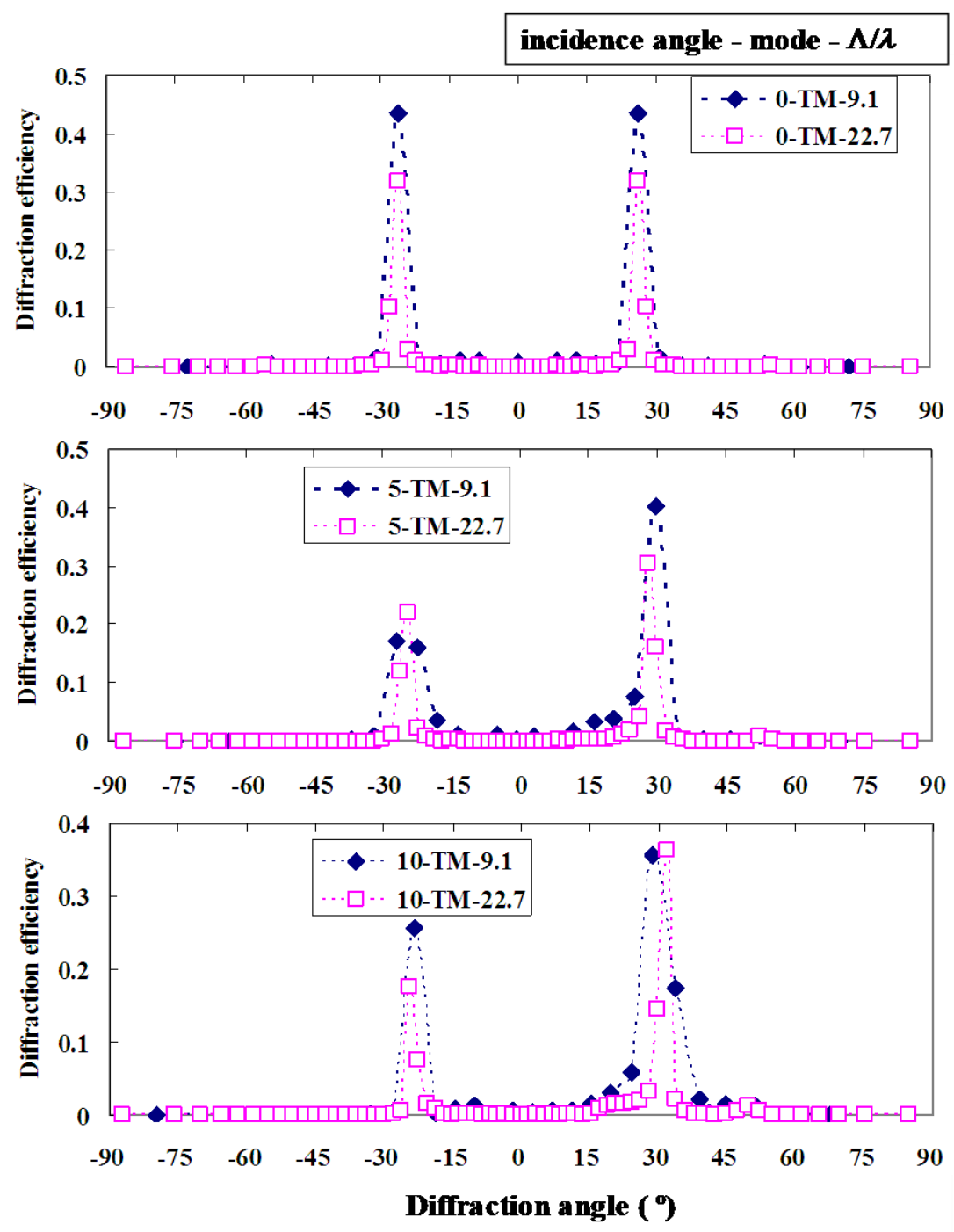

Fig. 9. Angular transmissivity spectra of the triangular grating for TM mode as a function of the wavelength and incidence angle in case A. Graph legend: light incidence angle, mode of polarization, value of $\Lambda / \lambda$, respectively. 
Table 1. $\Delta \theta_{\text {average }}$ of the three shapes for different polarization and direction of incident light.

\begin{tabular}{|c|c|c|c|c|c|}
\hline Shape & S-Triangle & Rectangle & Sine & A-Triangle-s & A-Triangle-o \\
\hline TE-A & $1.72^{\circ}$ & $7.48^{\circ}$ & $2.83^{\circ}$ & $0.84^{\circ}$ & $10.85^{\circ}$ \\
\hline TE-B & $2.36^{\circ}$ & $27.35^{\circ}$ & $14.81^{\circ}$ & $15.23^{\circ}$ & $16.03^{\circ}$ \\
\hline TM-A & $1.72^{\circ}$ & $2.54^{\circ}$ & $2.82^{\circ}$ & $0.85^{\circ}$ & $10.85^{\circ}$ \\
\hline TM-B & $4.34^{\circ}$ & $5.02^{\circ}$ & $2.96^{\circ}$ & $11.52^{\circ}$ & $20.67^{\circ}$ \\
\hline
\end{tabular}

S-triangle: symmetric triangle

A-Triangle-s: asymmetric triangle with the same light direction

A-Triangle-o: asymmetric triangle with the opposite light direction 